\title{
Empiric evidence for a genetic contribution to predisposition to surgical site infection
}

\author{
James P. Lee, MD' ${ }^{1}$, Harriet W. Hopf, MD ${ }^{1}$, and Lisa A. Cannon-Albright, PhD $^{2,3}$ \\ ${ }^{1}$ Department of Anesthesiology, University of Utah School of Medicine \\ ${ }^{2}$ Division of Genetic Epidemiology, Department of Medicine, University of Utah School of \\ Medicine \\ ${ }^{3}$ George E. Wahlen Department of Veterans Affairs Medical Center, Salt Lake City, Utah. Division \\ of Genetic Epidemiology, Department of Medicine, University of Utah School of Medicine
}

\begin{abstract}
The genetics of microbial pathogens have been extensively studied, but there has been little work on human genetic susceptibility to surgical site infection (SSI). We analyzed a large genealogical population database to study the familial contribution to SSI.

We analyzed 651 individuals with ICD-9 codes indicating presence of SSI. Matched hospital controls were randomly selected from the database based on birth year, sex and birthplace. The average relatedness of all possible pairs of cases, and separately of controls (x1000 sets) was compared empirically. The relative risk for SSI was estimated by comparing the number of observed affected individuals among the relatives of cases to the number of affected individuals observed among relatives of matched hospital controls. The Genealogical Index of Familiality test for patients with SSI showed significant excess relatedness ( $p<0.010)$; this excess was still observed when close relationships were ignored $(\mathrm{p}=0.019)$. The $\mathrm{RR}$ for third-degree relatives of cases was significantly elevated $(1.62, p=0.029)$. The significant excess relatedness and the significantly elevated RR to distant relatives support a genetic predisposition to acquiring SSI.
\end{abstract}

\section{Keywords}

surgical site infection; genetics; familiality; population database

\section{Introduction}

\begin{abstract}
Surgical site infection (SSI) and other healthcare-associated infections (sepsis, pneumonia, urinary tract infection) remain the most common complications of surgery, and are associated with increased length of stay, cost, and mortality ${ }^{1}$. Many risk factors increase the risk of $\mathrm{SSI}^{2}$. Some of these risk factors are related to the procedure itself ${ }^{2-4}$ and some are specific to the patient ${ }^{2,5}$. Procedural risks include surgical technique, degree of contamination at the operative site, duration of the operation, site of operation, skin antisepsis, surgical drains, operating room ventilation, duration of surgical scrub, and preoperative skin preparation ${ }^{2}$. Patient-specific risk factors associated with SSI include
\end{abstract}

Corresponding Author: Harriet W. Hopf, University of Utah School of Medicine, 3C444, 30 N 1900 E, Salt Lake City, UT 84132, Phone: 801-205-1013, Fax: 801-581-4367, Harriet.hopf@ hsc.utah.edu.

Preliminary results were presented at the Wound Healing Society Annual Meeting in Orlando, FL, April 2010. Final results presented at the International Anesthesia Research Society meeting in Vancouver, BC, May 2011.

Conflict of Interest: None of the authors reports any financial relationships that may pose a conflict of interest. 
wound hypoxia, age, sex, diabetes mellitus, obesity, smoking, malnutrition, duration of hospital stay, cardiopulmonary dysfunction, and immunosuppression ${ }^{2}$.

Although the genetics of the microbes that cause disease have been extensively studied, little research has evaluated host (human) genetic predisposition to SSI. Personalized medicine is the concept of using individual genetic information to customize individual patient management. ${ }^{6}$ Single nucleotide polymorphisms (SNP) are stable genetic variations in base pairs present in a population with $>1 \%$ frequency. SNPs provide much of the genetic basis for disease ${ }^{7}$. Personalized medicine is most advanced in the area of pharmacogenomics, where, for example, warfarin dosing can be predicted by genotype ${ }^{8}$. We postulate that there may be genetic variants that predict susceptibility to wound infection.

In order to evaluate whether there is a genetic susceptibility to SSI, we used a unique population-based resource consisting of a genealogy linked to medical data in Utah. We examined the genetic relationships among individuals identified in this Utah Population Database who also had evidence of being diagnosed with a SSI. Using two well-established and published methods, the Genealogical Index of Familiality and estimation of relative risks in relatives, we explored the hypothesis of a heritable predisposition to SSI.

\section{Materials and Methods}

\section{The Utah Population Database (UPDB)}

The Utah Population Database contains genealogical information for the Utah pioneer founders and over 2 million of their descendants 9 . The UPDB includes over 9 million individuals, most without genealogy data. We specifically selected those individuals for study who have both parents, all 4 grandparents, and at least 6 of their 8 great grandparents included in the UPDB. These strict criteria for genealogic data allowed us to match cases and controls with similar amounts of genealogy data.

Since 1994, all of the patient information from inpatient hospital and outpatient clinical visits from the University of Utah Health Sciences Center (UUHSC-serving 20\% of Utah) has been stored in a data warehouse ${ }^{10-13}$. UUHSC patients have been record-linked to the Utah genealogy data so that the genetic relationships between individuals with a specific phenotype may be studied. The tools used to analyze these linked data allow for examination of numerous phenotypes and have been well reported ${ }^{10-13}$.

With the approval of both the oversight body of the Utah Population Database and the University of Utah institutional review board, we identified patients with diagnosed SSI based on ICD-9 codes. The U.S. Centers for Disease Control and Prevention defines a surgical site infection as: "an infection that occurs after surgery in the part of the body where the surgery took place. Surgical site infections can sometimes be superficial infections involving the skin only. Other surgical site infections are more serious and can involve tissues under the skin, organs, or implanted material."4 This definition is not clearly duplicated in the International Classification of Disease, Ninth Revision (ICD-9) diagnostic codes used for medical records.

A major challenge for our study was the variable ways in which SSI are captured in the medical record. We analyzed a number of combinations of ICD-9 codes to balance sample size and specificity. ICD-9 codes identified as potentially useful in identifying patients diagnosed with SSI are displayed in Table 1. Table 2 lists the combinations of case definitions used, the ICD codes for each group and the number of cases we identified for each group using the strict genealogy requirements previously described. 


\section{Genealogical Index of Familiality}

We used the Genealogical Index of Familiality (GIF) to test the hypothesis of no excess relatedness among affected individuals. This test was developed for the Utah Population Database $^{9}$. The GIF test measures the average relatedness of the set of patients of interest (cases) and compares that to the measure of expected average relatedness estimated in the UPDB. Unlike relative risk, the GIF allows evaluation of both close and distant relationships between individuals.

The Malécot coefficient of kinship ${ }^{14}$, defined as the probability that randomly selected homologous genes from two individuals are identical by descent from a common ancestor, is used to measure pairwise relatedness in the GIF. For a sibling pair the coefficient is 0.25 $\left(1 / 2^{2}\right)$, for a grandparent-grandchild pair it is $0.125\left(1 / 2^{3}\right)$, for a first cousin pair 0.0625 $\left(1 / 2^{4}\right)$, and so on. Pairs of individuals with a larger genetic distance have a smaller average relatedness measure.

The case-Genealogical Index of Familiality is defined as the average of the coefficients of kinship between all possible pairs of affected individuals $\left(\mathrm{X} 10^{5}\right)$. The control-Genealogical Index of Familiality is the average of the coefficients of kinship between all possible pairs in a set of matched control individuals. The pairwise relatedness of the patients is compared with the distribution of the average pairwise relatedness statistics estimated for 1000 independent sets of matched controls, providing an empirical test of significance. Controls must be appropriately matched to cases for factors that might be associated with relatedness in Utah. The UUHSC provided us with the identification numbers of $20 \%$ of all UUHSC hospital patients, representing each sex, birth year and birthplace cohort, for use as controls. Each case and each potential control was assigned to one of 132 cohorts based on sex, 5year birth year group, and birthplace (Utah or not). Cohort-matched controls were randomly selected from this set of hospital patients using a random number generator.

Due to the observation of both close and distant relationships, the overall GIF test is only a test of excess relatedness. Excess relatedness might be observed in the presence of only genetic factors, in the presence of only familial factors (e.g., shared environment), or in the presence of a combination of both genetic and familial factors. We have therefore extended the GIF test to include a comparison of excess relatedness that ignores close relationships (first and second-degree relatives); we term this the distant GIF (dGIF) test ${ }^{9}$. This allows us to test the hypothesis that significant excess relatedness is observed among distant relatives. Since distant relatives are not expected to share environmental risk factors any more than population-level sharing, observed excess relatedness supports the presence of predisposing genetic factors.

\section{Relative Risks in Relatives}

Relative risks for relatives of SSI cases were estimated by comparing the number of observed affected individuals among the relatives of those individuals to the expected number of affected individuals in the UPDB based on internal rates. The expected number of affected individuals was estimated by selecting matched hospital controls and counting the affected relatives of those controls who were also hospital patients. We randomly selected a hospital matched control for each case and then counted the number of affected relatives of each one of the matched controls without duplication; we repeated this 100 times for each case. Relative risk was estimated among relatives of the case individuals as a classic casecontrol relative risk. A Fisher exact test for a $2 \times 2$ table was used to determine the significance of the test of the null hypothesis relative risk $=1.0$. Estimations of the confidence intervals for the relative risk were performed using the Agresti method. ${ }^{15}$ All statistical analysis was performed using software that was developed by investigators in the 
Division of Genetic Epidemiology / Department of Medicine at the University of Utah for the purpose of analysis involving the Utah Population Database and as described in previous studies 9 .

\section{Results}

\section{Genealogical Index of Familiality}

Table 3 shows the overall GIF and the distant GIF results for the 3 different groups of SSI cases considered. The table displays the number of individuals affected, their average relatedness (overall case GIF), the mean overall control relatedness from the analysis of 1000 sets of matched controls (mean control GIF), the empirical $p$ value of the comparison for all relationships (p), and the empirical $p$ value for the distant GIF test (dGIF p).

When we consider only the 350 SSI cases coded as non-healing surgical wound we do not see significant evidence for excess relatedness (Figure 1a). When our SSI definition is expanded from non-healing surgical wound to include prosthesis related infection or inflammation ( $\mathrm{n}=651)$, the average relatedness increases ( 4.62 to 5.53) and there is significant evidence for excess relatedness when all relationships are considered, and also when all close relationships are ignored (Figure 1b). Finally when we expand our group again (Figure 1c), and now include postoperative infection/central venous catheter or other infusion $(n=1650)$, the case average relatedness falls to 5.04 and we observe overall significant excess relatedness, but our evidence for excess distant relatedness is not significant $(\mathrm{p}=0.059)$.

\section{Relative Risk}

Table 4 shows the estimated RRs for the first-, second-, and third-degree relatives of cases in each surgical site infection group. Significantly elevated RRs were not observed for first-, second-, or third-degree relatives of SSI cases defined by "non-healing surgical wound" only. When we expanded the group definition to include prosthesis, although all the estimated $\mathrm{RR}$ were $>1.0$, only the third-degree relative risks were significantly elevated (RR $=1.62, \mathrm{p}=0.03)$. Finally, when we further expanded the definition to include infections related to catheters or infusion, all the RR were $>1.0$ and RRs for second- and third-degree relatives were significantly elevated.

\section{Discussion}

We report evidence for a familial/genetic contribution to SSI. We have attempted to identify $\mathrm{SSI}^{6}$ cases using 3 sequentially defined sets of codes, each more inclusive than the last. It is clear that the phenotype description is critical, although we saw similar results for all 3 phenotypes analyzed. The GIF analysis for each of the 3 defined groups of patients with SSI shows that the average relatedness observed for cases was significantly higher than expected. Only the group of SSI patients defined with inclusion of prosthesis, but not catheter/infusion showed significant excess clustering for all relationships and also for distant relationships only, although the other two SSI phenotype groups had similar dGIF (borderline significant) results, and the GIF distributions in Figures 1 show excess distant relationships for all 3 phenotype groups. As the sample size of each group increased, more relationships were observed and a smoother overall distribution for cases was observed. The GIF analysis of the largest set of SSI cases support a genetic contribution to predisposition to infection following surgery, with an excess of relationships even beyond third cousins (genetic distance $=8$ ).

The RR analysis for the 3 phenotype groups of SSI patients showed varying results as well. For the smallest phenotype group $(n=350)$, no first- or second-degree relatives were 
observed among relatives of matched controls. In the largest phenotype group considered $(\mathrm{n}=1650)$, in which many more relatives were considered, all of the RR estimates were $>1.0$ and the second- and third-degree RR estimates were significantly elevated. Again, these results support a shared genetic component to SSI predisposition.

One major limitation to this study is the use of ICD-9 codes to identify patients diagnosed with SSI. Physicians use variable codes to record SSI and surveillance for SSI is often inadequate ${ }^{16}$. Patients may develop SSI after discharge. UUHSC participates in the National Surgical Quality Improvement Project and the aggressive surveillance required by the program may have improved capture of SSI for more recent years. Although it is likely our data understate the incidence of SSI, it is unlikely that infections are not captured in a way that would bias our results. The reduced sample size entailed by missed SSI may have limited our ability to identify significant results. Some patients may have been inaccurately coded as having SSI and the codes we used would have captured some patients who did not in fact have SSI. Again, it is unlikely that there was a bias in how these data were included and excluded that would have changed our results. Because we are using a Utah population resource for analysis, SSI patients whose genealogy data is not complete in the UPDB and individuals diagnosed with a SSI before UUHSC data availability are also censored. We do not anticipate that this censoring results in any bias as it applies equally to all individuals whether they are cases or controls. Within the context of the goal of our study, our data support the idea that evaluation of individuals within affected pedigrees to determine which genes contribute to susceptibility to SSI is likely to be a fruitful avenue of research.

Another limitation of our study is the inability to control for confounding factors such as diabetes, obesity, smoking, need for multiple operations, need for higher risk operations, malnutrition, cardiopulmonary dysfunction, and use of immunosuppressant drugs. Some of these confounders have or may have a genetic basis, which could contribute to our findings.

Although the use of ICD-9 codes is limitation of our study, the data are of sufficient quality to support further investigation into the genetics of SSI susceptibility. The etiology of increased predisposition to SSI demonstrated in this study is likely both genetic and environmental. Our study does not define the genes that increase SSI risk. Likely candidates for a predisposition to SSI include SNPs related to impaired inflammation. Genes related to inflammation that have known SNPs include TNF-alpha, IL-1, IL-6, IL-10, heat shock genes, and lipopolysaccharide binding protein. ${ }^{7,17}$ Certain alleles of these genes are associated with greater risk of sepsis and / or of death from sepsis. Sepsis and SSI are clearly different disease processes, but inflammation plays a major role in each, so it is reasonable to speculate that these SNPs are also pertinent to SSI. Another candidate gene is the phagosomal oxidase (phox), which converts oxygen to superoxide, and plays a key role in host defense ${ }^{18}$. SNPs in phox cause Chronic Granulomatous Disease ${ }^{19}$, a rare condition that manifests early in childhood and in which patients develop severe recurrent infections, often of the skin, because of the inability to generate superoxide. The genetics of these catastrophic mutations have been studied in detail, but other genetic variations in phox have not been evaluated. It is plausible that SNPs that only reduce superoxide production may cause susceptibility to SSI without overt disease. Other genetic variants could predispose to disorders that require surgery or a propensity towards obesity, diabetes, connective tissue disorders, or other risk factors for SSI.

Our data support a genetic contribution to susceptibility to SSI. The next step is to define which genes are involved. Using the UPDB and with appropriate IRB approval we plan to identify, recruit, and obtain blood samples from members of the high-risk pedigrees identified in these analyses. This will allow us to genotype the most informative pedigrees 
and use linkage analysis as well as sequencing to identify candidate genes that may predispose patients to SSI and other infections.

\section{Acknowledgments}

L-C-A acknowledges support from the Huntsman Cancer Foundation.

Partial support for all data sets with in the Utah Population Database (UPDB) was provided by Huntsman Cancer Institute, University of Utah and the Huntsman Cancer Institute's Cancer Center Support grant, P30 CA42014 from National Cancer Institute.

National Library of Medicine grant LM009331 (to Lisa Cannon Albright)

\section{References}

1. Klevens RM, Edwards JR, Richards CL Jr, et al. Estimating health care-associated infections and deaths in U.S. hospitals, 2002. Public Health Rep. 2007; 122:160-6. [PubMed: 17357358]

2. Uckay I, Harbarth S, Peter R, Lew D, Hoffmeyer P, Pittet D. Preventing surgical site infections. Expert Rev Anti Infect Ther. 2010; 8:657-70. [PubMed: 20521894]

3. Hollenbeak CS, Lave JR, Zeddies T, Pei Y, Roland CE, Sun EF. Factors associated with risk of surgical wound infections. Am J Med Qual. 2006; 21:29S-34S. [PubMed: 17077416]

4. Mangram AJ, Horan TC, Pearson ML, Silver LC, Jarvis WR. Guideline for prevention of surgical site infection, 1999. Hospital Infection Control Practices Advisory Committee. Infect Control Hosp Epidemiol. 1999; 20:250-78. [PubMed: 10219875]

5. Hopf HW, Hunt TK, West JM, et al. Wound tissue oxygen tension predicts the risk of wound infection in surgical patients. Arch Surg. 1997; 132:997-1004. [PubMed: 9301613]

6. Piquette-Miller M, Grant DM. The art and science of personalized medicine. Clin Pharmacol Ther. 2007; 81:311-5. [PubMed: 17339856]

7. Hopf HW. Molecular diagnostics of injury and repair responses in critical illness: what is the future of "monitoring" in the intensive care unit? Crit Care Med. 2003; 31:S518-23. [PubMed: 12907881]

8. Finkelman BS, Gage BF, Johnson JA, Brensinger CM, Kimmel SE. Genetic warfarin dosing: tables versus algorithms. J Am Coll Cardiol. 2011; 57:612-8. [PubMed: 21272753]

9. Cannon Albright LA. Utah family-based analysis: past, present and future. Hum Hered. 2008; 65:209-20. [PubMed: 18073491]

10. Albright FS, Orlando P, Pavia AT, Jackson GG, Cannon Albright LA. Evidence for a heritable predisposition to death due to influenza. J Infect Dis. 2008; 197:18-24. [PubMed: 18171280]

11. Teerlink CC, Hegewald MJ, Cannon-Albright LA. A genealogical assessment of heritable predisposition to asthma mortality. Am J Respir Crit Care Med. 2007; 176:865-70. [PubMed: 17690335]

12. Weires MB, Tausch B, Haug PJ, Edwards CQ, Wetter T, Cannon-Albright LA. Familiality of diabetes mellitus. Exp Clin Endocrinol Diabetes. 2007; 115:634-40. [PubMed: 18058597]

13. Tashjian RZ, Farnham JM, Albright FS, Teerlink CC, Cannon-Albright LA. Evidence for an inherited predisposition contributing to the risk for rotator cuff disease. J Bone Joint Surg Am. 2009; 91:1136-42. [PubMed: 19411462]

14. Malecot, G. Les Mathematiques de L'Heredite. Paris: Masson; 1948.

15. Agresti, A. Categorical Data Analysis. New York: WIley; 1990.

16. Barnes S, Salemi C, Fithian D, et al. An enhanced benchmark for prosthetic joint replacement infection rates. Am J Infect Control. 2006; 34:669-72. [PubMed: 17161743]

17. Vodovotz Y, Constantine G, Faeder J, et al. Translational systems approaches to the biology of inflammation and healing. Immunopharmacol Immunotoxicol. 2010; 32:181-95. [PubMed: 20170421]

18. Nauseef WM. Assembly of the phagocyte NADPH oxidase. Histochem Cell Biol. 2004; 122:27791. [PubMed: 15293055] 
19. Stasia MJ, Li XJ. Genetics and immunopathology of chronic granulomatous disease. Semin Immunopathol. 2008; 30:209-35. [PubMed: 18509647]

\author{
Abbreviations \\ GIF Genealogical Index of Familiality \\ ICD 9 International Classification of Disease, Ninth Revision \\ RR Relative Risk \\ SSI Surgical Site Infection \\ UPDB Utah Population Database \\ UUHSC University of Utah Health Sciences Center
}



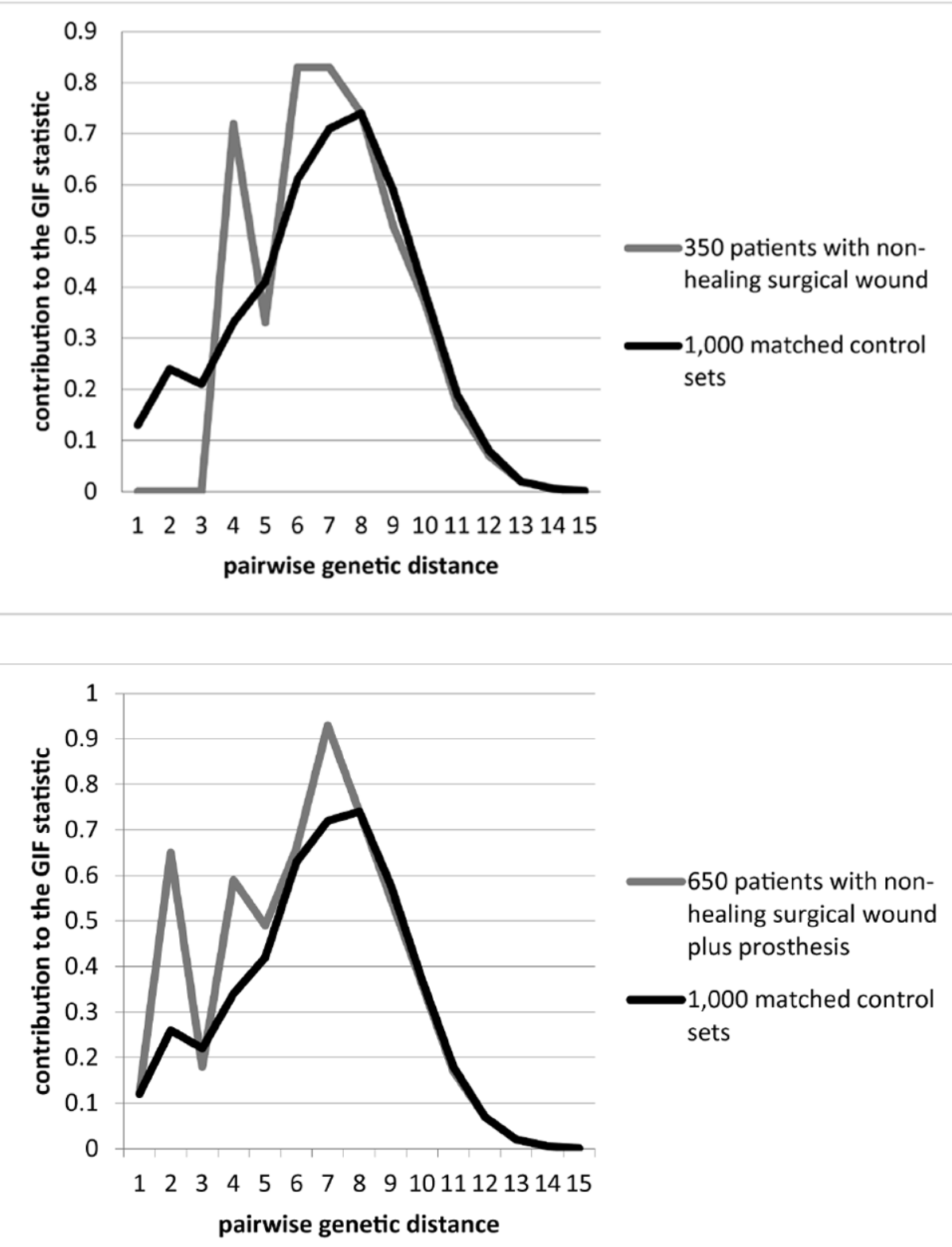

Wound Repair Regen. Author manuscript; available in PMC 2014 March 01. 


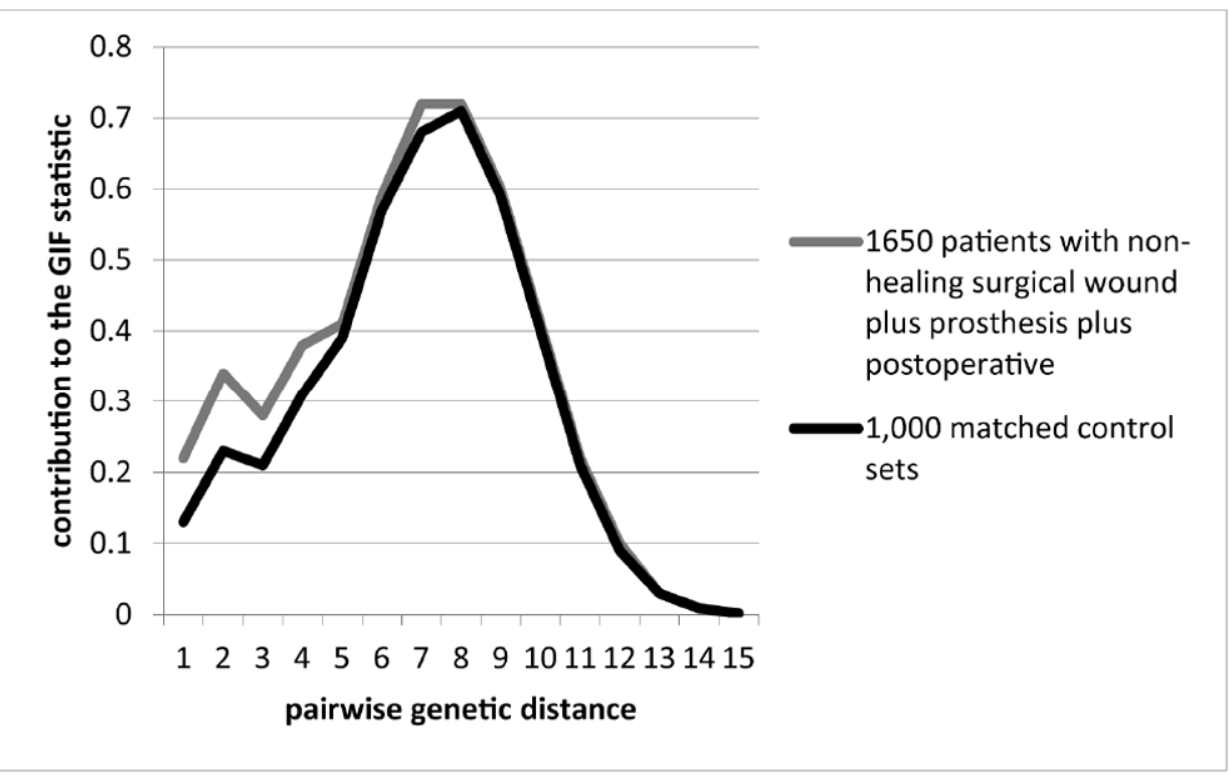

Figure 1.

a. Contribution to the GIF statistic by genetic distance Light line 350 Surgical Site Infection Cases

Dark line 1,000 sets of matched controls

b. Contribution to the GIF statistic by genetic distance

Light line 651 Surgical Site Infection Cases with prosthesis

Dark line 1,000 sets of matched controls

c. Contribution to the GIF statistic by genetic distance

Light line 1,650 Surgical Site Infection Cases with prosthesis/catheter/infusion

Dark line 1,000 sets of matched controls 
Table 1

ICD-9 Codes Considered for Analysis

\begin{tabular}{|c|c|}
\hline ICD-9 Code & Definition \\
\hline 998.83 & Non-healing surgical wound \\
\hline 996.66 & Infection and inflammatory reaction; due to internal joint prosthesis \\
\hline 996.67 & Infection and inflammatory reaction; due to other internal orthopedic device, implant and graft, Internal fixation device \\
\hline 998.3 & Disruption of operation wound \\
\hline 998.5 & Other and unspecified infection due to central venous catheter \\
\hline 999.31 & Infection following other infusion, injection, transfusion, or vaccination \\
\hline 999.39 & Mediastinitis \\
\hline $519.2^{*}$ & Disruption of Caesarean wound \\
\hline $674.1 *$ & \\
\hline
\end{tabular}

* Note that codes 674/1 and 519.2 did not appear in the database, so these codes could not be used for analysis. 
Table 2

Combinations of Codes Used for Analysis

\begin{tabular}{|c|c|c|}
\hline ICD-9 Codes & Definition & $\begin{array}{c}\text { Number of Patients in } \\
\text { Cohort }\end{array}$ \\
\hline 998.83 & Surgical wound complications only & 350 \\
\hline $998.83 ; 996.66-7$ & Surgical wound complications + infections related to joint replacements & 651 \\
\hline $\begin{array}{c}998.3 ; 998.83 ; 996.66-7 ; 998.5 ; \\
999.31 ; 999.39\end{array}$ & $\begin{array}{c}\text { Wound complications + infections in joint replacements + other } \\
\text { infections related to interventions }\end{array}$ & 1650 \\
\hline
\end{tabular}




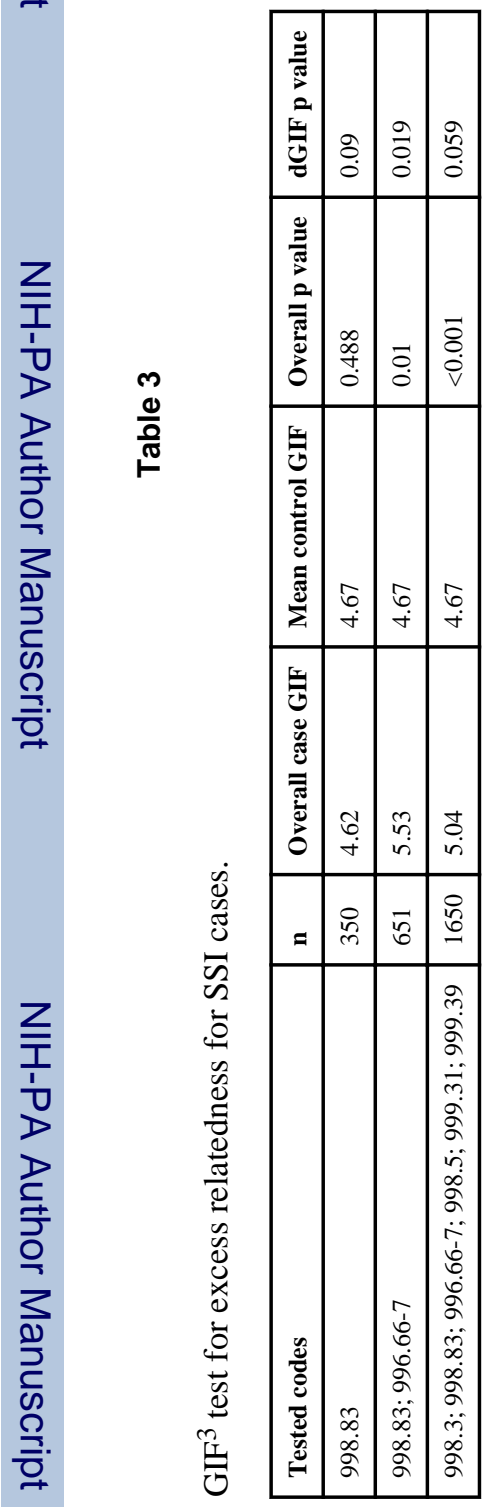

Wound Repair Regen. Author manuscript; available in PMC 2014 March 01. 


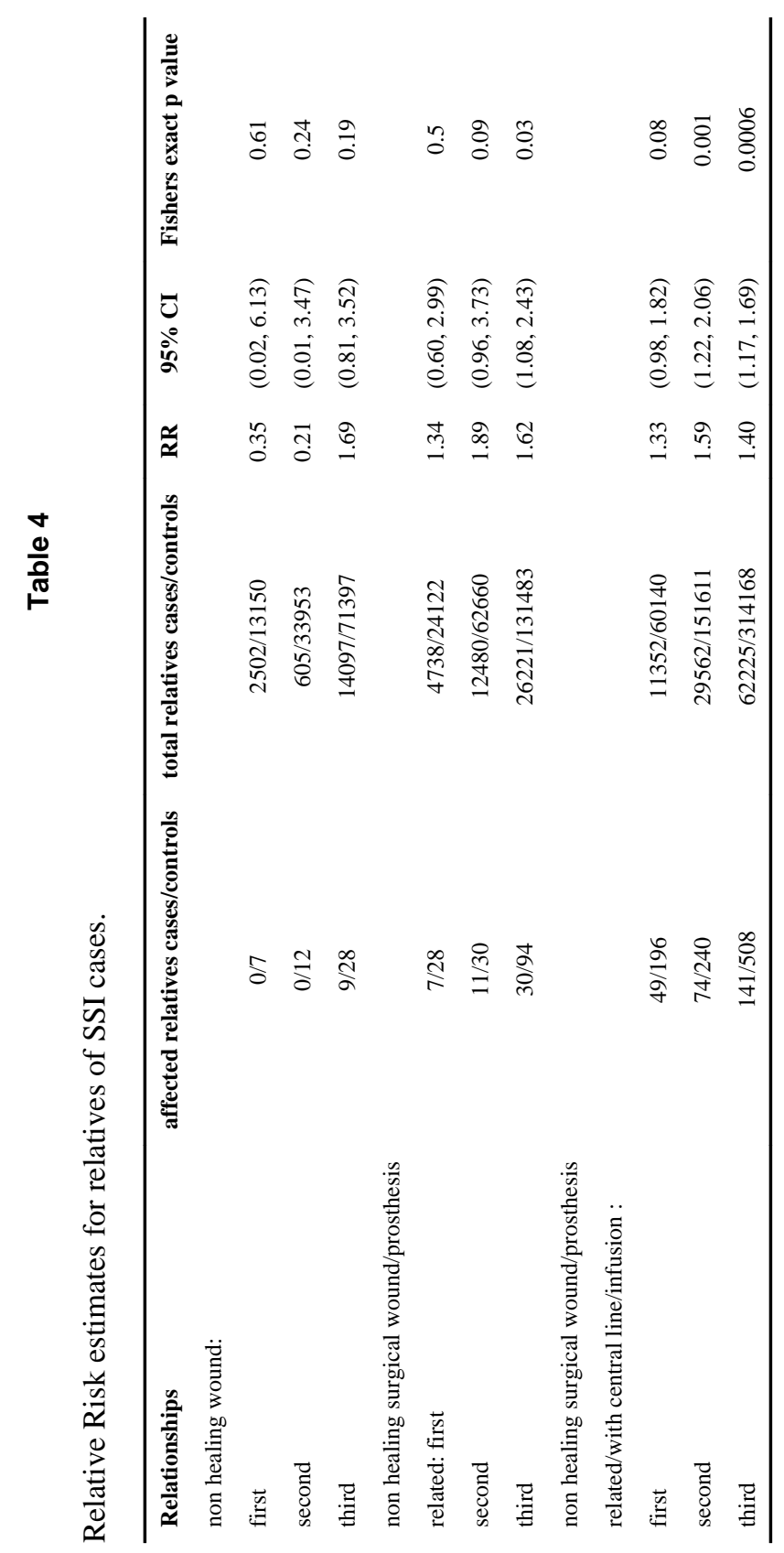

Wound Repair Regen. Author manuscript; available in PMC 2014 March 01. 